\title{
Complication of Hollow Organ Perforation after Radical Nephrectomy in Wilms Tumor: A Case Report
}

\author{
Helmy Fahada', Fransiska Kusumawidagdo ${ }^{2}$ \\ ${ }^{1}$ Department of Surgery, Faculty of Medicine, Universitas Airlangga \\ Dr. Soetomo General Hospital, Surabaya, Indonesia \\ ${ }^{2}$ Department of Pediatric Surgery, Faculty of Medicine, Universitas Airlangga \\ Dr. Soetomo General Hospital, Surabaya, Indonesia
}

*Corresponding author details: Helmy Fahada, M.D.; helmysurgeon@gmail.com

\begin{abstract}
The most frequent kidney cancer in children is Wilms tumor, often known as nephroblastoma. The prevalence is low and only representing for approximately $7 \%$ of all childhood cancers. The most standard treatment for this tumor is surgery. Complications from surgical care occurred in patients with Wilms tumor who had a radical nephrectomy. Intestinal blockage, hemorrhage, infection, and vascular injury are the most common surgical consequences. The purpose of this case report is to describe the incidence of perforation of hollow organs after radical nephrectomy in the case of Wilms tumor in a child aged 2 years and 6 months after neoadjuvant chemotherapy, who later developed generalized peritonitis due to ileal anastomoses leakage.
\end{abstract}

\section{Keywords: Wilms tumor; radical nephrectomy; complication; hollow organ perforation}

\section{INTRODUCTION}

Wilms tumor is the most frequent type of kidney cancer in children. Wilms tumor affects roughly 8 out of every million children under the age of 15 in the United States [1]. Wilms tumor is responsible for $7 \%$ of all cancers in children. In North America, an estimated 500 new cases of Wilms tumor are diagnosed each year. Almost two-thirds of Wilms tumor cases are diagnosed in children under the age of five, and 95 percent are diagnosed before the kid reaches the age of ten. Currently, there was no Wilms tumor epidemiological investigation in Indonesia. [2]

Multimodal therapies are used to treat Wilms tumor patients in the majority of cases $[3,4]$. Surgery, chemotherapy, and radiation are administered in stages, which can boost 5-year survival rates by up to $90 \%$. Treatment options are determined by the stage of the Wilms tumor. Since the 1970 s, experts have recommended preoperative radiation and chemotherapy. Adjuvant radiation and chemotherapy are avoided in children with a very low risk of recurrence and metastasis. The surgeon will choose surgery as the primary treatment option as long as the tumor is operable [5].

In this case report, the author describes the occurrence of hollow organ perforation as a complication following radical nephrectomy in children aged 2 years and 6 months with Wilms tumor.

\section{CASE PRESENTATION}

A female child aged 2 years 6 months came to the pediatric polyclinic of Dr. Soetomo General Hospital (Surabaya, Indonesia) with main complaints of enlarged stomach which was known by his parents in the past 2 weeks. The child did not complain of abdominal pain. In the last two weeks the lump was getting bigger, therefore the patient's parents brought the patient for treatment.

On the left side of the abdomen, there was an enlarged bulge that was not painful.
In other areas, no swollen glands or tumors were discovered. Another complaint was that this patient had a mild temperature for the previous week but was nevertheless fairly active. Since the last three weeks, the patient's urine had been reddish in hue.

Other family members did not have similar problems, according to the patient's family history. The patient is the first kid of a Javanese married couple. None of the patient's relatives, whether children or adults, were found to have malignancies or cancer. During her pregnancy, the patient's mother checked in with the local midwife on a regular basis and was never diagnosed with a pregnancy condition. The patient was born normally, crying immediately, and weighing 3400 grams at birth. Patients' basic immunization records are comprehensive, including Hepatitis B, Polio, DPT, HiB, Measles, and MMR. The patient received breast milk until he was two years old, after which he switched to formula milk. Since the age of six months, the patient had received complementary meals in the form of instant porridge and team rice since the age of one year. The patient was able to lift his head at the age of three months, sit up at the age of six months, walk at the age of twelve months, and communicate fluently at the age of eighteen months. When compared to their peers, the patient's parents did not report any developmental delays. The child appears fully conscious on physical inspection. Vital signs included a blood pressure of 90/60, a pulse of 130 times/minutes, a respiratory rate of 36 times/minutes, and a body temperature of $36.8^{\circ} \mathrm{C}$. There were no abnormalities found on examination of the head, neck, thorax, or extremities. The bowel sounds were normal, the liver and spleen were not palpable, and the abdominal veins were prominent. There was $15 \times 15 \mathrm{~cm}$ mass on the left abdomen, well-defined, painless, nonmobile at the base but moveable at the surface. The mass was solid and pliable, and the outside skin appears normal, not yellowish. The existence of this tumor had a significant impact on the activities of children. 
During admission for hospitalization, the patient underwent several laboratory tests: hemoglobin 10.8 $\mathrm{g} / \mathrm{dL}$, leucocytes $21,370 / \mathrm{mm} 3$, platelets $239,200 / \mathrm{mm} 3$. We also performed serum electrolytes test: sodium 135 $\mathrm{mmol} / \mathrm{L}$, potassium $3.5 \mathrm{mmol} / \mathrm{L}$, and chloride $96 \mathrm{mmol} / \mathrm{L}$. Plain radiographs of the thoracic and abdominal regions are showed in Figure 1.
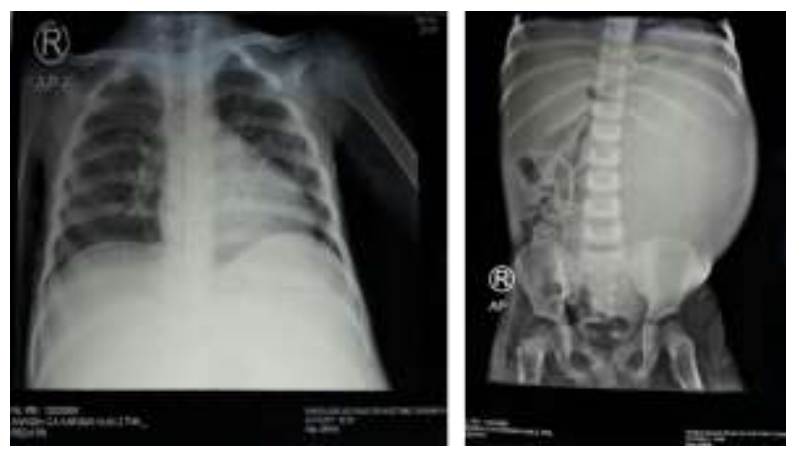

FIGURE 1: Plain Photo Radiological Examination

With the help of a radiologist, an abdominal ultrasound examination was performed on this patient (Figure 2), which revealed a left middle abdomen solid tumor measuring $20 \times 15 \mathrm{~cm}$ was considered to be malignant, with ascites and potential infiltration of the pancreas and spleen. The suspected origin of this tumor is from the left kidney (suspected Wilms Tumor). The liver, right kidney, and bladder all appeared to be in good condition.
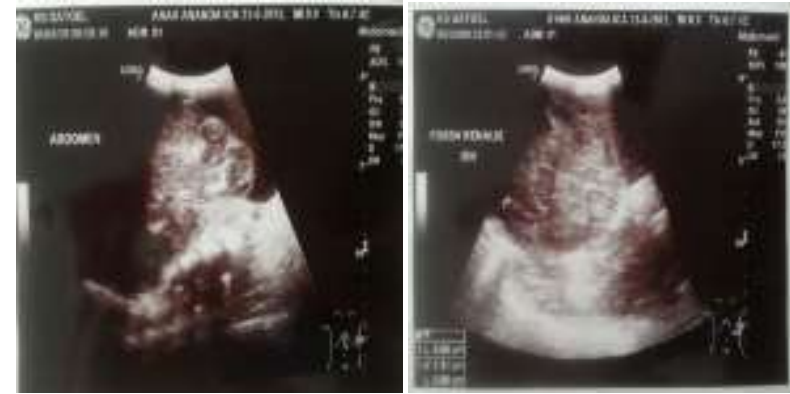

FIGURE 2: Abdominal Ultrasonography

A contrast abdominal CT scan was performed on the patient to more about the tumor's genesis and resectability potential (Figure 3). The radiologists' expertise on the CT scans performed were: In the kidney, there was a mixed solid mass (38 HU), cystic (20 HU), and calcification (133 HU) mass with well-defined, uneven margins, and a size of $+12.5 \times 14.4 \times 18.5 \mathrm{~cm}$, which displayed heterogeneous contrast enhancement on the solid and cystic walls (50HU). The mass appeared to extend and cross the midline, pushing the abdominal aorta to the right posterolateral side, the bowel structure to the left antero-lateral side, extending into the pelvic cavity and pushing the bladder to the inferior side with clear boundaries, and there was no picture of a normal left kidney. The left renal artery looked to be the source of blood supply for the tumor. The inferior vena cava to the left kidney had a thrombus (the length of the thrombus was $\pm 5.6 \mathrm{~cm}$, and it filled the lumen to \pm 99 percent of the total lumen). The radiologists' conclusions were mixed cystic lesion with calcified component in the left kidney, size $\pm 12.5 \times 14.4 \mathrm{x}$ $18.5 \mathrm{~cm}$ with the above-mentioned extension supports the Wilms tumor picture.
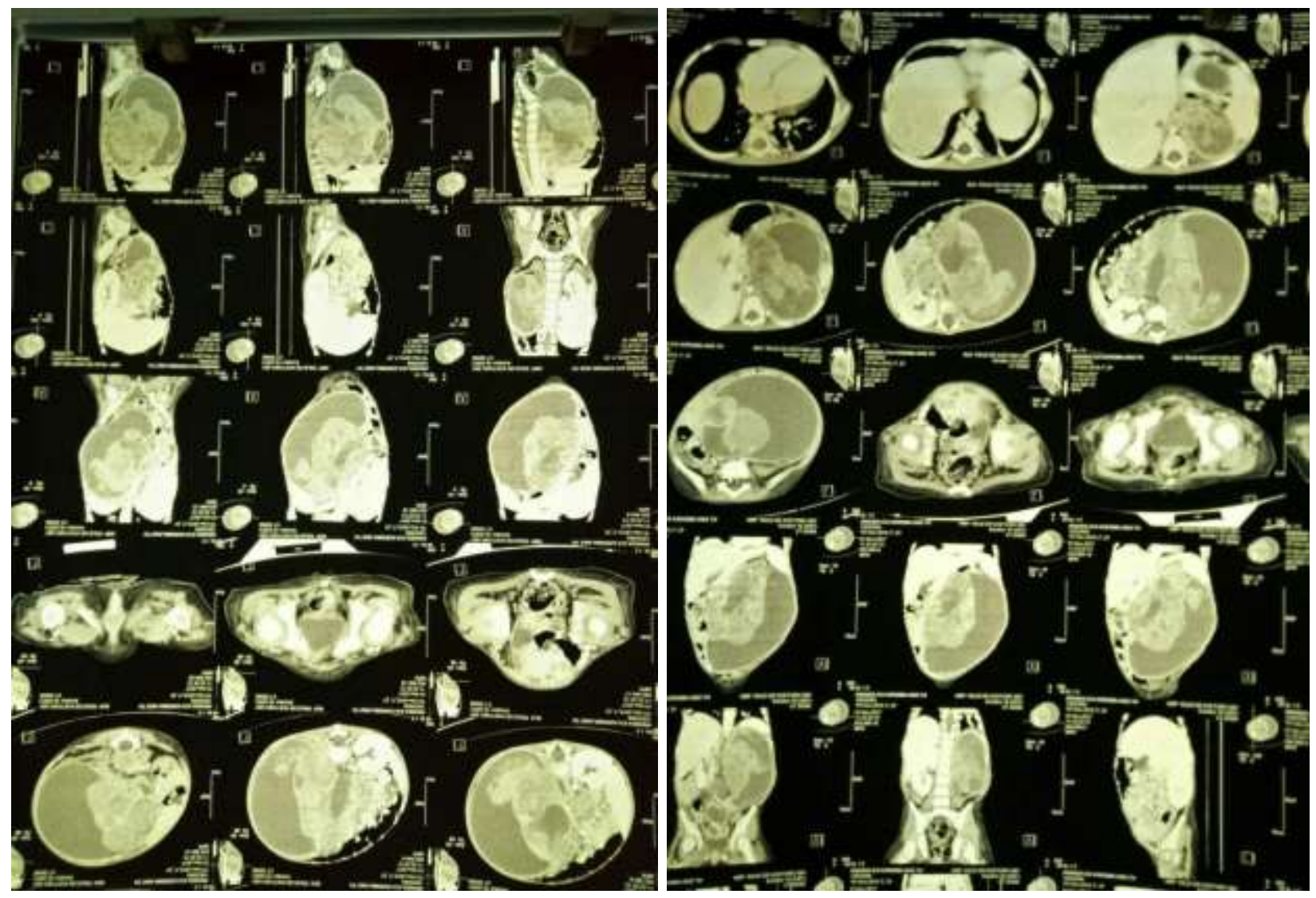

FIGURE 3: Abdominal CT scan with contrast 
The diagnosis was established based on clinical examination obtained with imaging studies that supported the presence of Wilms tumor in the left kidney. The patient was scheduled to have 3 weeks of Neoadjuvant Chemotherapy with Actinomicyne-D and Vincristine before undergoing radical left kidney resection (left radical nephrectomy).

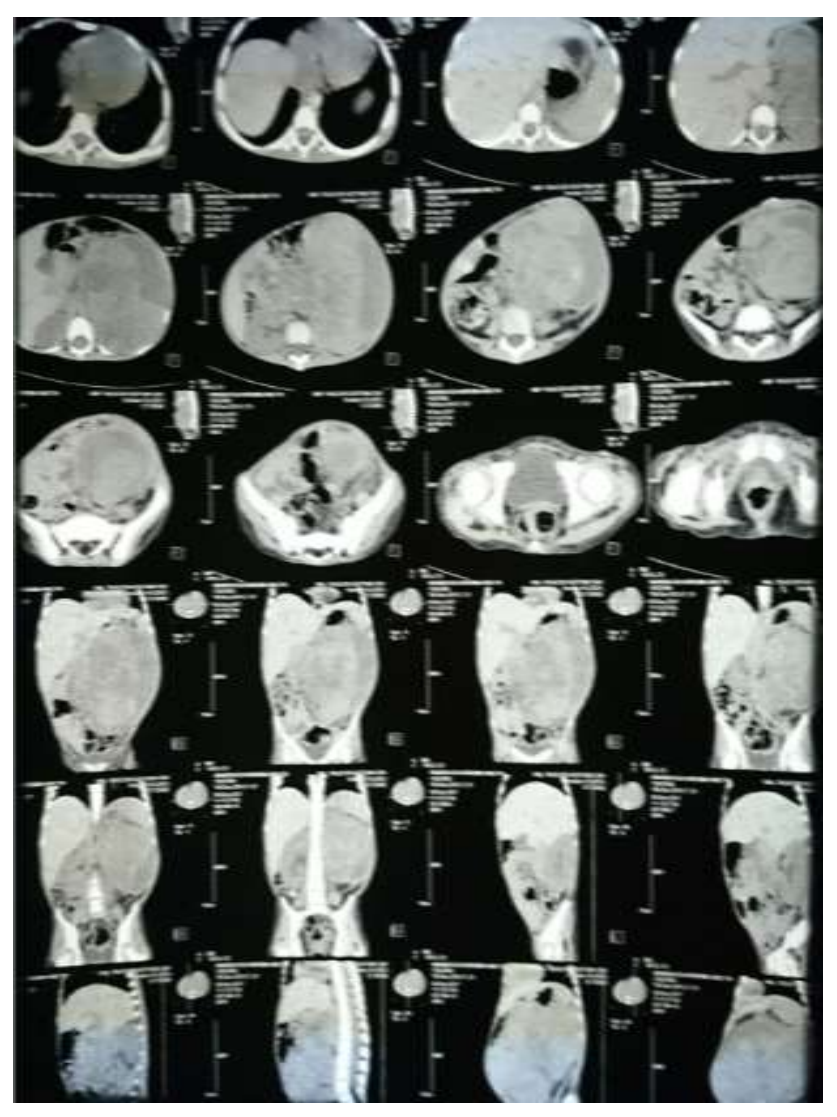

After undergoing neoadjuvant chemotherapy (before surgery), the patient underwent a re-evaluation of CT scan with contrast to measure the tumor and its expansion (Figure 4). The following were the findings of the CT Scan expertised evaluation: Fixed cystic lesions with calcified components in the left Renal with a dimension of $\pm 12.5 \mathrm{x}$ $11.3 \times 15.5 \mathrm{~cm}$, with this growth corroborating the Wilms' tumor picture.

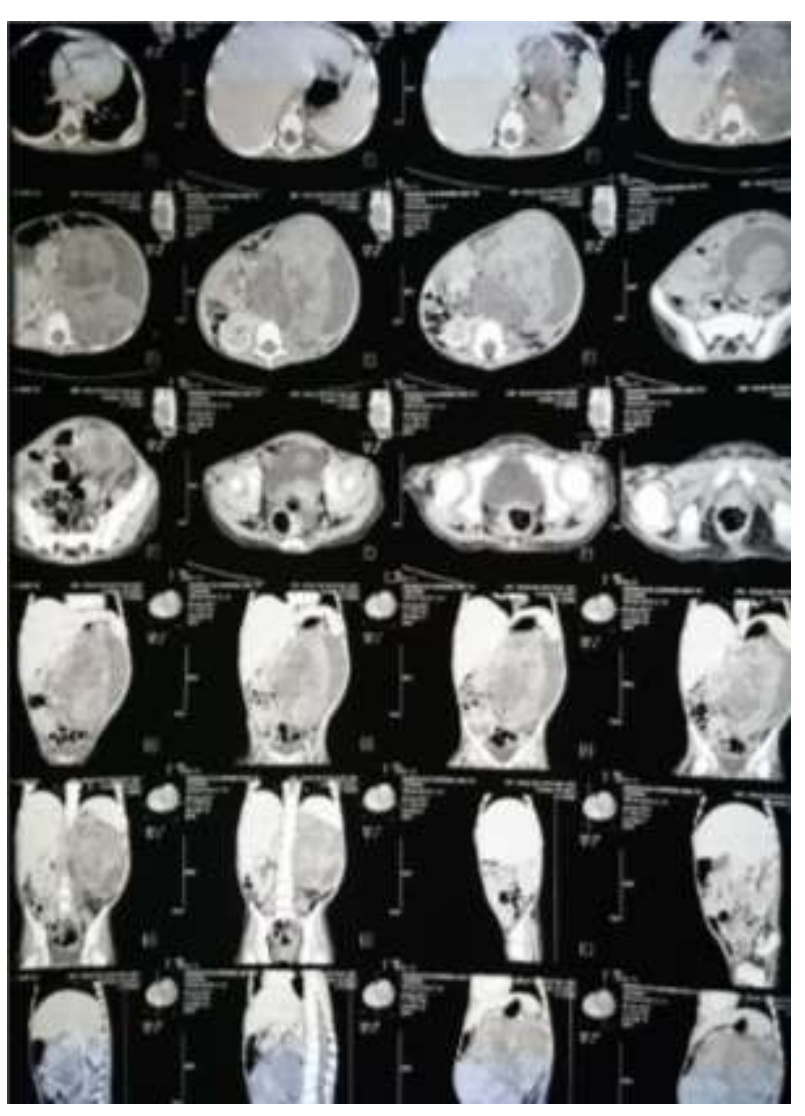

FIGURE 4: Abdominal CT scan with contrast after neoadjuvant chemotherapy

After neoadjuvant treatment, the tumor had shrunk to a manageable size. This patient was still planned for radical nephrectomy to provide definitive therapy. A laparotomy was done on the patient, after which the tumor was removed and radical nephrectomy was performed. The operation discovered During the operation, an $18 \times 13 \times 7 \mathrm{~cm}$ solid cystic tumor mass attached to the surrounding tissue. The transverse colon was attached to the tumor mass and its \pm 12 $\mathrm{cm}$ long of mesocolon. Multiple nodules were also found around the renal vasa and ureter with the largest size located in the ureter, which is $3 \times 1 \times 1 \mathrm{~cm}$.

The tumor mass was separated from the surrounding tissue during this procedure. There was only a small amount of cloudy yellow tumor fluid leaking. The kidney was removed in a radical nephrectomy procedure. A portion of the capsule was firmly attached to the colon's outer wall, while the remaining capsule was liberated as much as possible. In addition, several nodules in the renal arteries were removed. This patient had roughly $500 \mathrm{cc}$ of bleeding.

The patient was given antibiotics Cefotaxime + Sulbactam and Metronidazole after surgery to address infections in the urinary system (leukocytes 21,370, accompanied by hematuria). This patient had infection problems while receiving surgical care. A positive ESBL blood culture was found in this patient. Two weeks following the first procedure, monitoring in the patient's room revealed evidence of hollow organ perforation (Figure 5). During the postoperative treatment of radical nephrectomy, this patient also had electrolyte imbalance. To aid healing, this patient was given electrolyte and albumin preparations.
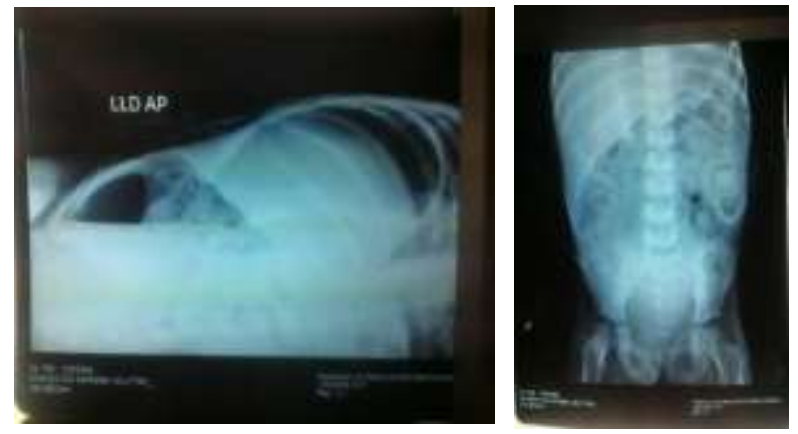

FIGURE 5: Plain radiograph of the abdomen in 2 positions indicates free air in the abdominal cavity

This patient underwent a second procedure, an exploratory laparotomy and repair of the perforation site, after establishing a perforation in a hollow organ. Multiple perforations with hazy peritoneal fluid mixed with feces equivalent to roughly $300 \mathrm{cc}$ occurred during the operation. The duodenum, jejuno-ileal, and colon all had perforations: duodenum pars 4 was perforated with a diameter of $2 \mathrm{~mm}$; the descending colon distal to the splenic flexure was perforated with a diameter of $3 \mathrm{~cm}$; perforations with diameters from $2 \mathrm{~mm}-1 \mathrm{~cm}$ were found at $15 \mathrm{~cm}, 30 \mathrm{~cm}, 50$ $\mathrm{cm}, 105 \mathrm{~cm}, 110 \mathrm{~cm}, 115 \mathrm{~cm}$ from the Treitz ligament; 100 $\mathrm{cm}$ from the Treitz ligament discovered minor tissue; and a laceration was found at 70 and $75 \mathrm{~cm}$ from the Treitz ligament. 
At $70 \mathrm{~cm}$ from the Treitz ligament, this patient had a $5-\mathrm{cm}$ ileal resection, end-to-end anastomosis, primary repair of the other perforation, and a loop ileostomy at $10 \mathrm{~cm}$ proximal to the ileocecal junction. The patient was treated with abdominal cavity washing until it's clear, adhesiolysis, and the placement of a drain in the Douglas cavity.

The patient developed abdominal cavity abnormalities five days following the second surgery, including widespread peritonitis due to anastomotic leakage from the ileum (Figure 6). A third operation was performed on the patient to manage the infection's source. During the investigation, faecal material was discovered along with murky peritoneal fluid. Anatomical leakage was also discovered at 20,50, and $70 \mathrm{~cm}$ from the Treitz ligament. After basic repair, the patient's abdominal cavity was cleansed and a bogota bag was implanted. According to the peritoneal fluid culture in the third operation, the patient's antibiotic was switched to Meropenem.

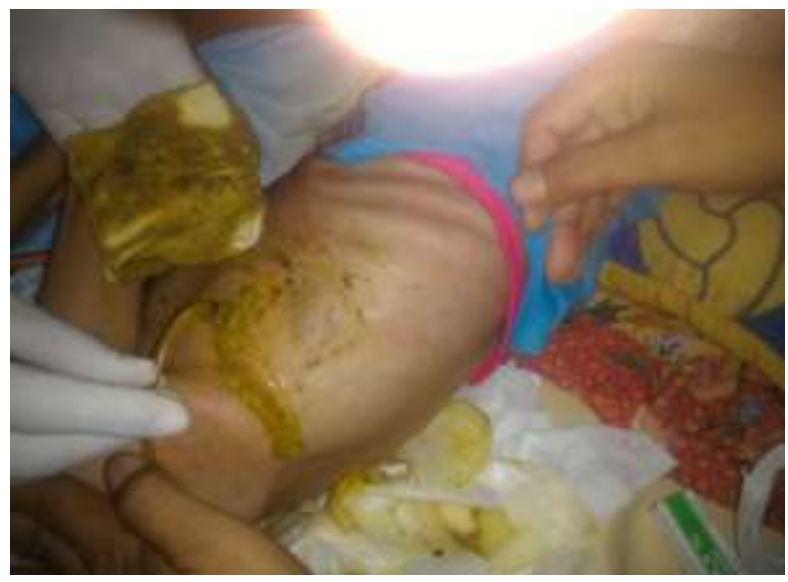

FIGURE 6: Clinical picture of the patient when there is anastomotic leakage, it shows spillage in the form of feces coming out of the drain tube of Douglas Cavitiy.

\section{DISCUSSION}

This patient case report fits the clinical picture of a Wilms tumor. In terms of age and imaging evaluation, both the clinical presentation and the imaging examination appeared to be normal. To lessen the size of the tumor, the patient was given neoadjuvant chemotherapy in the hopes that the surgeon would not have any complications during the operation. Although the tumor size was reduced, the reduction was not significant. The SIOP protocol was used in this patient, which included preoperative chemotherapy to reduce tumor size, reduce the danger of tumor rupture during surgery, and lower the chance of local and distant recurrence [6].

The "gold standard" of preoperative chemotherapy in Wilms' tumor, according to SIOP, is vincristine and actinomycin D for 4 weeks. In this case vincristine and actinomycin D were given for 3 weeks $[6,7]$.

During the radical nephrectomy procedure, adhesions were discovered, and the tumor was quite large. Tissue trauma could occur when the surrounding tissue is freed, especially where the tumor is firmly attached, like in this example at the colon and jejunoileal. As a result, the risk of perforation in these patients is quite high. Wilms' tumor in this patient was stage 2 according to the SIOP staging system, one of which was owing to invasion of surrounding organs but was still entirely resectable.

After about 3 weeks, there was a perforation in the hollow organ with the discovery of free air in the abdominal cavity. This perforation ass thought to be caused by a big tumor that is attached to the surrounding tissue.
A second operation was performed on this patient, which included an exploratory laparotomy and the repair of several perforation locations using end-to-end anastomosis procedures on the ileum and primary suture on the remaining perforation sites.

Neutropenia enterocolitis is an inflammatory bowel disease that affects immunocompromised patients, particularly those receiving chemotherapy. The fundamental feature of this disease is the occurrence of intestinal mucosal damage, which occurs in conjunction with neutropenia and a weakened immune system in the patient. Inflammatory circumstances can lead to intestinal perforation in neutropenic enterocolitis. Taxane, cytosine arabinoside, gemcitabine, vincristine, doxorubicin, gemcitabine, cyclophosphamide, 5-fluorouracil, leuvocorin, and daunorubicin are some of the chemotherapy medicines used to treat this condition. Patients who have a neutrophil count of less than 500/L are at a greater risk of developing neutropenic enterocolitis. Based on the report, this case was found in this patient with a neutrophil count $<1000 / \mu \mathrm{L}$ [8]. The patient in this case underwent vincristine chemotherapy for 3 weeks, which according to Rodriguez (2017) the chemotherapy drug is one of the agents suspected of causing neutropenic enterocolitis. However, the patient's neutrophil count was found to be high or normal based on the findings of the type of leukocyte calculation during hospitalization, ruling out the possibility of organ perforation in the patient due to neutropenic enterocolitis [8].

The patient experienced anastomotic leakage and peritonitis 5 days after having an exploratory laparotomy and having perforated hollow organs repaired. The intraabdominal healing process of this patient was not good considering the occurrence of ESBL infection and the nutritional condition of this patient was quite poor. The failure of the wound healing process resulted in re-leakage of the segment of intestinal anastomosis. Peritonitis developed as a result of this procedure. While performing primary repairs on the leaking segment, an exploratory laparotomy was performed again and the abdominal cavity was washed. To avoid the abdominal wound not closing properly, the patient was implanted with a bogota bag. According to the latest culture results, the patient's antibiotics were switched to meropenem.

There are several complications of radical nephrectomy surgery in cases of Wilms tumor. Complications occurred in 12.7 percent of Wilms tumor patients who had a radical nephrectomy. The most common consequences, according to the study, were intestinal obstruction (5.1\%), extensive bleeding (1.9\%), surgical site infection (1.9\%), and vascular injury (1.9\%). (1.5 percent). The greater the cancer's spread, the higher the danger of problems [5]. The patient in this case had a perforation of the hollow organ after radical nephrectomy, which according to the study was not a common surgical complication in Wilms tumor cases.

\section{CONFLICT OF INTEREST}

The authors declared no competing interest.

\section{REFERENCES}

[1] C. W. Zuppan, J. B. Beckwith, and D. W. Luckey, "Anaplasia in unilateral Wilms' tumor: a report from the National Wilms' Tumor Study Pathology Center," Hum Pathol, vol. 19, pp. 1199-209, Oct 1988.

[2] [2] A. Grovas, A. Fremgen, A. Rauck, F. B. Ruymann, C. L. Hutchinson, D. P. Winchester, et al., "The National Cancer Data Base report on patterns of childhood cancers in the United States," Cancer, vol. 80, pp. 232132, Dec 151997. 
[3] J. J. Li, H. H. Huang, J. Shen, J. Y. Jiang, F. Pan, S. T. Yu, et al., "Multimodal therapy for adult Wilms' tumour: an experience from one centre," Clin Transl Oncol, vol. 13, pp. 672-6, Sep 2011.

[4] D. K. Nakayama and P. C. Bonasso, "The History of Multimodal Treatment of Wilms' Tumor," The American Surgeon, vol. 82, pp. 487-492, 2016.

[5] Ehrlich P.F. and S. R.C, Ashcraft's Pediatric Surgery - 6th Edition: Chapter 65 Renal Tumors: Elsevier Saunders: London.
[6] A. Groenendijk, H. van Tinteren, Y. Jiang, R. R. de Krijger, G. M. Vujanic, J. Godzinski, et al., "Outcome of SIOP patients with low- or intermediate-risk Wilms tumour relapsing after initial vincristine and actinomycin-D therapy only - the SIOP 93-01 and 2001 protocols," Eur J Cancer, vol. 163, pp. 88-97, Jan 152022.

[7] D. M. Green, C. A. Cotton, M. Malogolowkin, N. E. Breslow, E. Perlman, J. Miser, et al., "Treatment of Wilms tumor relapsing after initial treatment with vincristine and actinomycin D: a report from the National Wilms Tumor Study Group," Pediatr Blood Cancer, vol. 48, pp. 493-9, May 2007.

[8] F. G. Rodrigues, G. Dasilva, and S. D. Wexner, "Neutropenic enterocolitis," World J Gastroenterol, vol. 23, pp. 42-47, Jan 72017. 